\title{
Post-operative Myelopathy (Paraplegia), Unusual Causes to Consider - Case Reports
}

\section{Forshing Lui ${ }^{1 *}$, Ning Zhong ${ }^{2}$, John Geraghty ${ }^{3}$, Zoe Robinow ${ }^{1}$ and Kathleen Barnett ${ }^{1}$}

${ }^{1}$ California Northstate University College of Medicine, Elk Grove, CA, USA

${ }^{2}$ Kaiser Permanente Medical Center, Sacramento, CA, USA

${ }^{3}$ Kaiser Permanente Medical Center, Roseville, CA, USA

*Corresponding Author: Forshing Lui, California Northstate University College of Medicine, Elk Grove, CA, USA.
Received: August 24, 2021

Published: September 18, 2021

(C) All rights are reserved by Forshing Lui., et al.

\section{Abstract}

Post-operative paraparesis due to spinal cord pathology (myelopathy) is an uncommon yet devastating complication related to surgery or anesthesia. Direct causes are either related to the spinal column or the procedure such as spinal fractures, spondylosis, rheumatoid and other spondyloarthropathies, spinal osteomyelitis, discitis, epidural abscess, and spinal tumors. These causes of post-operative paraparesis are more obvious and often anticipated pre-operatively. We reported two uncommon causes which needs to be considered with a high index of suspicion. These are spinal cord infarction related to aortic manipulations or interventions, intraoperative hypotension, use of vasoconstrictors with the anesthetics, or epidural anesthesia. The typical clinical presentation is acute (wake up from surgery and anesthesia) onset severe paraparesis due to an acute ischemic myelopathy. The diagnostic MRI findings are vertebral body infarction in addition to the abnormal spinal cord signal. The other rare yet important cause is functional vitamin B12 deficiency induced by use of nitrous oxide anesthesia. The diagnostic findings are high plasma methylmalonate level and the classical MRI findings of abnormal signals in the dorsal columns (the inverted V sign).

Keywords: Myelopathy; Spinal Cord Infarction; Vertebral Body Infarction; Nitrous Oxide; Subacute Combined Degeneration; Dorsal Column; Inverted V Sign

\section{Abbreviations}

EHL: Extensor Hallucis Longus; S2-S5: Sacral 2 to Sacral 5 Levels; ED: Emergency Department; CSF: Cerebrospinal Fluid; WBC: White Blood Cell Count; ESR: Erythrocyte Sedimentation Rate; CRP: C-Reactive Protein; ANA: Antinuclear Antibody; MCV: Mean Corpuscular Volume

\section{Introduction}

As surgeons, it is most disheartening to operate on a patient with a very well-performed procedure to see the patient waking up with paraparesis. There are many known causes including all types of spinal surgeries with underlying spinal pathology including fractures, spondylosis, rheumatoid and other spondyloarthropathies, spinal osteomyelitis, discitis, epidural abscess, and spinal tumors. These causes of post-operative paraparesis are more obvious and often anticipated pre-operatively. There are also causes related to anesthetic procedures such as hematoma following spinal or epidural procedures. We are describing two uncommon cases of spinal cord pathology (myelopathy) not caused by direct spinal procedure, yet the patients may present with post-operative myelopathy. A high index of suspicion is most important for arriving at the correct diagnosis by the surgeon or consulting neurologist. 


\section{Case One}

A 50-year-old obese male who noted exhaustion and burning in his legs several hours after riding his stationary bicycle. The symptoms resolved spontaneously in the evening. The next morning, he felt stiff and went to stretch when he suddenly felt intense burning of both thighs, scrotum, and penis radiating down both legs. He developed leg weakness, inability to ambulate without assistance, and bowel and bladder incontinence. He was sent to the emergency department.

Neurologic examination revealed $4+/ 5$ strength in his hip extensors, 4/5 right tibialis anterior, 5/5 left tibialis anterior, 4/5 bilateral EHL, 3/5 right plantar flexors, and 4/5 left plantar flexors. Otherwise, strength of other muscles was 5/5 with normal tone. Reflexes were 2+ in the arms and knees with absent ankle reflexes and flexor plantar responses bilaterally. There was decreased pinprick sensation in the left S2-S5 region but intact on the right. Vibration sense was impaired in the great toes bilaterally.

CSF analysis performed in the ED showed protein $50 \mathrm{mg} / \mathrm{dL}$, glucose $75 \mathrm{mg} / \mathrm{dL}$, and WBC 4/mL. Serologies were negative for infectious etiology or heavy metal toxicity. Inflammatory markers including ESR, CRP, and ANA and a hypercoagulable workup were within normal limits except for mildly elevated protein C.

A lumbar spine MRI with and without gadolinium showed T2 hyperintensity changes in the conus medullaris (CM) and L2 vertebral body with enhancement of the cauda equina (CE) and CM (Figure 1 and 2).

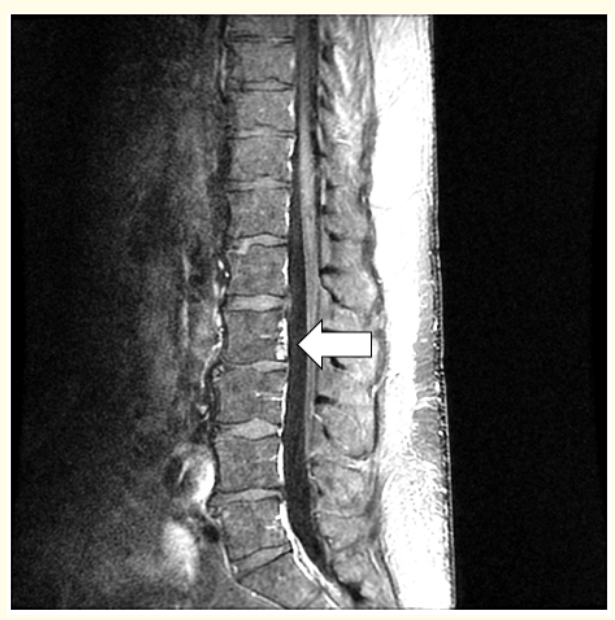

Figure 1: T1 showing infarct of L2 vertebral body.

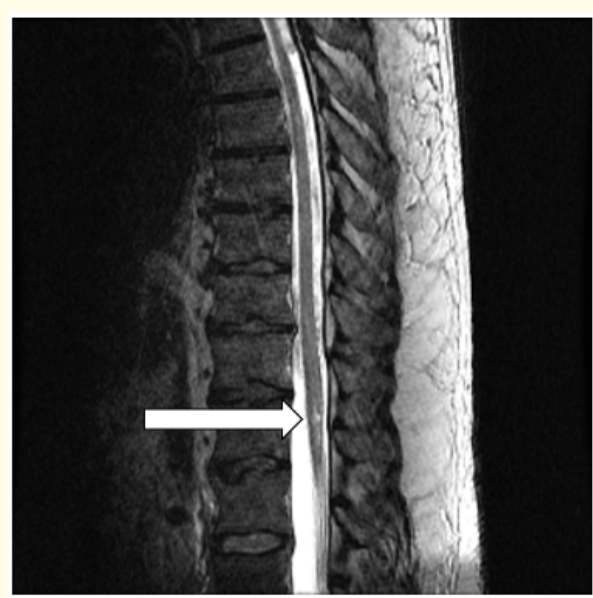

Figure 2: STIR image showing signal change in the terminal cord and $\mathrm{T} 2$ hyperintensity in the conus medullaris.

The final diagnosis is spontaneous spinal cord (conus medularis) infarction. The patient received high-dose steroids while hospitalized without clinical benefit. His strength slowly improved over several months, especially in his left leg. He has persistent sensory impairment in his buttocks, scrotum, and plantar surfaces of both feet. He continues to experience bowel and bladder incontinence.

\section{Case Two}

A 24-year-old male patient presented with lower extremity numbness and tingling for one month. The symptoms progressively worsened. He then developed lower extremity weakness and gait difficulty for about 2 weeks. He felt dizzy and unsteady when closing his eyes. He did fall a few times because he could not feel his feet. He has noted that increased difficulty with urination and bowl movement.

He has good past health. There was no history of sick contact or recent travel.

Socially, he does not smoke or drink yet he does use "whippits", about 15 cannisters a day for several months.

Physical exam was significant for abnormalities mainly in his nervous system. He had grade 4/5 weakness distally affecting his 
hands and grade 4/5 affecting both hip flexors and ankle dorsiflexors. Other muscles showed normal strength. His deep tendon reflexes were 1+ all over with indeterminate plantar response. His sensory examination was significant for Sensation, decreased to light touch, pinprick below bilateral wrist, diminished vibration sensation up to bilateral knee level, abnormal proprioception in toes. Coordination was normal with finger to nose and heel to shin testing. His gait was broad-based with positive Rombergism. He was unable to walk on feels and he failed tandem gait.

His lab exam showed a normal complete blood count with normal MCV. His vitamin B12 level is within normal range at $220 \mathrm{pg} /$ $\mathrm{mL}$ (normal $200-600 \mathrm{pg} / \mathrm{mL}$ ). His methylmalonate level was high at $1.4 \mathrm{umol} / \mathrm{L}$ (normal <= $0.3 \mathrm{umol} / \mathrm{L}$ ). His MRI spine showed an extensive $\mathrm{T} 2$ signal hyperintensity from $\mathrm{C} 2$ to $\mathrm{T} 2$ affecting mainly the dorsal columns with an inverted V sign (Figure 3).

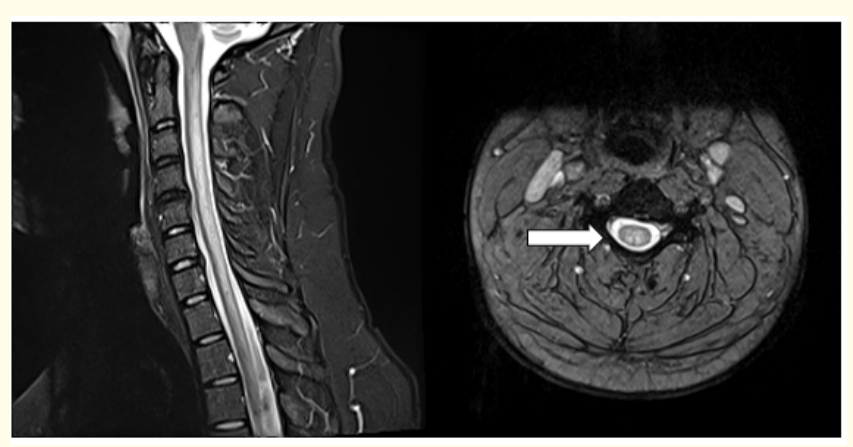

Figure 3: Long segment of T2 hyperintensity in the spinal cord from C2 to T2, affecting mainly the dorsal columns with an "inverted V sign".

The final diagnosis is subacute combined degeneration of the spinal cord caused/precipitated by nitrous oxide abuse. He was advised to stop using "whippits" and given intramuscular followed on discharge with oral vitamin B12. His symptoms stabilized and gradually improved so he was able to walk more steadily without any obvious weakness after 3 months.

\section{Discussion}

Both these two reported cases are not caused directly by surgery or anesthesia. However, both cases may be the result of sur- gical/interventional procedure involving the aorta, or use of epidural, or nitrous oxide anesthesia.

Spontaneous spinal cord infarctions are infrequent. It is estimated that 1.6 - 7.2 spinal cord infarctions occur per 100,000 individuals yearly, with a slightly higher occurrence in females than males [1]. Spinal cord infarction after anesthesia and surgery have been reported more often yet still very infrequent [2-4]. The reported pathophysiological mechanisms include spinal cord ischemia following epidural anesthesia associated with intraoperative hypotension, vasospasm associated with use of epinephrine-containing anesthetics, or toxicity from local anesthesia. Spinal cord infarction occurs more commonly following aortic procedures including repair of aortic aneurysms, aortic dissection, repair of arteriovenous malformations, and interventional arterial procedures through the aorta [5]. The more important question is how to make or confirm the diagnosis of spinal cord infarction. As shown in our case, the important clinical clues will include acute onset (usual presentation post-op, when the patient awakens from anesthesia), paraparesis with a sensory level and relative paring of the dorsal column sensation. The most important imaging modality is MRI of the spine with abnormal T2 signal inside the spinal cord. The presence of abnormal signal involving the vertebral body suggestive of vertebral body infarction essentially clinches the diagnosis [6].

Vitamin B12 deficiency can cause megaloblastic anemia. In some patients, it causes subacute combined degeneration of the spinal cord with specific degeneration of the dorsal column and lateral columns. Dorsal column involvement is usually more evident presenting clinically with severe sensory ataxia due to loss of proprioception and vibratory sensation. The lateral column or corticospinal tract involvement can lead to spasticity, weakness, and upgoing plantar response on examination. The classical MRI change is an inverted $\mathrm{V}$ sign $[7,8]$. Our patient has a normal vitamin B12 level. He suffers from a functional vitamin B2 deficiency due to his history of nitrous oxide (whippits) abuse [9,10]. Similar clinical scenario can occur in a patient who wakes up from a surgical procedure with nitrous oxide anesthesia [11]. Nitrous oxide oxidizes the cobalt ion of vitamin B12. The oxidized form does not function well as a coenzyme in the methionine synthase pathway. This leads to a functional deficiency of vitamin B12 despite a relatively normal blood level. This diagnosis needs to be considered in any patient who wakes up with paraparesis from a surgical procedure with nitrous oxide anesthesia. As shown in our case, the important 
diagnostic approaches include a high index of suspicion especially in any patient who had nitrous oxide anesthesia. Lab tests may confirm the diagnosis with complete blood counts showing high MCV, a low or low normal vitamin B12 level, and most important a high methylmalonate level confirming functional deficiency of vitamin B12 and activity of methionine synthase. MRI spine is helpful in defining spinal cord pathology especially when abnormal signal affects symmetrically bilateral dorsal columns, the inverted V sign. The presence of vertebral body infarction on MRI is diagnostic of a spinal cord infarction.

\section{Conclusion}

Post-operative paraparesis due to spinal cord pathology (myelopathy) is an uncommon yet devastating complication related to surgery or anesthesia. Direct causes are either related to the spinal column or the procedure such as spinal fractures, spondylosis, rheumatoid and other spondyloarthropathies, spinal osteomyelitis, discitis, epidural abscess, and spinal tumors. These causes of postoperative paraparesis are more obvious and often anticipated preoperatively. Two other uncommon yet important causes needs to be considered with a high index of suspicion. These are spinal cord infarction related to aortic manipulations or interventions, intraoperative hypotension, use of vasoconstrictors with the anesthetics, or epidural anesthesia. The diagnostic finding is vertebral body infarction in addition to the abnormal spinal cord signal. The other important cause is functional vitamin B12 deficiency induced by use of nitrous oxide anesthesia. The diagnostic findings are high plasma methylmalonate level and the classical MRI findings of an inverted $\mathrm{V}$ sign.

\section{Bibliography}

1. Qureshi AI., et al. "A Population-Based Study of the Incidence of Acute Spinal Cord Infarction". Journal of Vascular and Interventional Neurology 9.4 (2017): 44-48.

2. Weinberg L., et al. "Post-operative paraplegia following spinal cord infarction”. Acta Anaesthesiologica Scandinavica 46.4 (2002): 469-472.

3. Chan LL., et al. "Post-epidural analgesia spinal cord infarction: MRI correlation". Acta Neurologica Scandinavica 105.4 (2002): 344-348.
4. Kobayashi K., et al. "Spinal cord infarction following epidural and general anesthesia: a case report". JA Clinical Reports 3.1 (2017): 1-5.

5. Cheshire WP., et al. "Spinal cord infarction: etiology and outcome”. Neurology 47.2 (1996): 321-330.

6. Faig J., et al. "Vertebral body infarction as a confirmatory sign of spinal cord ischemic stroke: report of three cases and review of the literature". Stroke 29.1 (1998): 239-243.

7. Yuan JL., et al. "Nitrous oxide induced subacute combined degeneration with longitudinally extensive myelopathy with inverted V-sign on spinal MRI: a case report and literature review". BMC Neurology 17.1 (2017): 1-4.

8. Narra R., et al. "Inverted V sign" in sub-acute combined degeneration of cord". Journal of Clinical and Diagnostic Research: JCDR 9.5 (2015): TJ01.

9. Al-Sadawi M., et al. "Inhaled Nitrous Oxide 'Whip-Its!' Causing Subacute Combined Degeneration of Spinal Cord". American Journal of Medical Case Reports 6.12 (2018): 237.

10. Onrust MR and Frequin ST. "Subacute combined spinal cord degeneration by recreational laughing gas (N20) use". Journal of Central Nervous System Disease (2019).

11. Patel KK., et al. "Subacute combined degeneration of the spinal cord following nitrous oxide anesthesia: a systematic review of cases". Clinical Neurology and Neurosurgery 173 (2018): 163-168.

\section{Volume 4 Issue 10 October 2021}

(C) All rights are reserved by Forshing Lui., et al. 